\title{
Progress and Prospects in Magnetic Data Storage
}

\author{
Robert L. White \\ Center for Research on Information Storage Materials Department of Materials Science \& Engineering \\ Stanford University, Stanford. CA 94305
}

\begin{abstract}
Magnetic recording has been the dominant data storage technology for many years, and presently delivers higher bit density at lower cost than magneto-optic data storage. The trend of ever-increasing bit density for magnetic recording is now, however, encountering some fundamental physical barriers. We examine bere these barriers and conclude that a change in magnetic recording paradigm, probably including the use of patterned media, will be required to carry magnetic data storage to and beyond $100 \mathrm{Gbit} / \mathrm{in}^{2}$.
\end{abstract}

Key words: magnetic recording, bit density, granular media, patterned media

\section{Background}

The dominant data storage technology for many years has been magnetic recording. The capacity for data storage of magnetic media has increased at an astonishing rate in the last decade. The bit density of hard disk drives has shown an annual density growth rate of $30 \%$ per year from 1960 to 1990 and $60 \%$ per year since 1990 . Extrapolating these trends for another decade leads to data densities well in excess of $100 \mathrm{Gbit} / \mathrm{in}^{2}$, and the question "Can we get there with present technology?" is urgently being raised.

In the course of the last several decades, there have been a number of predictions on the data density ultimately achievable with magnetic recording technology. Some of those proposed "limits" were at the level of tens of Megabits/in", later "limits" were at hundreds of Megabits/in ${ }^{2}$. The industry has charged through all such "limits" with impunity. In the past year, laboratory demonstration at $5 \mathrm{Gbit}^{2} \mathrm{in}^{2}$ have been announced by IBM $^{13}$, by Fujitsu ${ }^{2)}$ and by Headway/HMT ${ }^{3)}$ and of 12 Gbits/in ${ }^{2}$ by IBM ${ }^{4}$. At these densities, however, one is beginning to encounter barriers raised by fundamental physical limits, not just human ingenuity, so it is valuable to look at magnetic data storage anew, and ask what technology or paradigm changes will be required to reach or exceed densities in the $100 \mathrm{Gbit} / \mathrm{in}^{2}$ range.

Throughout the recent era $(40 \mathrm{yrs})$ of magnetic data storage the overarching paradigm has remained unaltered. The medium is comprised of a thin film of small, independently switching, magnetic particles. A bit is written on the medium with an inductive ring head, with the fringing field at the gap doing the writing and with the bit shape and position determined by the magnetic fields and field gradients generated by the head. The magnetization is in the plane of the film (longitudinal), and one bit is composed of many $-10^{3}$ to $10^{5}$ - magnetic grains. The bits are read by detecting the fringing fields generated by the magnetization of the film. Up through the 70 's and into the 80 's, the magnetic storage medium employed small discrete particles of a magnetic oxide dispersed in a polymer binder. During the $1980^{\prime}$ 's the transition (for disk media) was made to thin metal (cobalt or cobalt alloy) polycrystalline films. The grain size in these films was and is typically much smaller than the size of the discrete oxide particles, and the increase in bit density achieved in the 80's and 90's was based on the capabilities of such thin film media. Until 1990, the read process was the mirror image of the write process, with the fringing fields generated by the medium inducing magnetization of the inductive head, detected in turn by pick-up coils. Since 1990 the inductive head has been increasingly supplanted in high performance systems by more sensitive magnetoresistive heads; the increase in slope of the bit density vs. time starting in 1990 is often attributed to the arrival of the MR head. Presently spinvalve heads, employing the giant magnetoresistance effect and having still higher sensitivity, are being introduced and will probably, in the next decade, become dominant in high density storage applications.

\section{Problems in Extending Bit Density}

As bit density gets higher, and bits get smaller, all system components of the data storage system become more difficult. The signal per bit is smaller. Tracking becomes more difficult. Flying height must be reduced, making tribological problems more acute. At present, however, the dominant factor limiting the increase in data density is media noise, in turn caused by the granularity of the medium. When bit size was much larger and the number of magnetic grains per bit was effectively infinite, grain size was not such a significant factor (though grains clumping into exchange-coupled clusters was and is). As bits get smaller and smaller, however, the granularity of the medium becomes increasingly a problem. Figure 1 shows an MFM image of recorded bits. At 5000 flux reversals/mm (bit length $2000 \AA$ ) the bits are clearly very ragged and not the crisp rectangles one would desire. This raggedness translates directly into signal/noise deterioration. In principle, the problem can be solved by going to smaller grains. This solution is blocked by the superparamagnetic effect, the loss of stability of the magnetization of a small particle due to thermal fluctuations. This magnetization decay effect has been the subject of much recent theory and experiment". The lifetime of the magnetization of a small particle is $M(t)=M(0) e^{-\frac{x w}{k T}}$ exponential in its total anisotropy energy. In this expression $\mathrm{Ku}$ is the uniaxtal anisotropy constant of the material, $V$ the particle volume, $k$ Boltzman's constant, and $\mathrm{T}$ the temperature. For a magnetization state lifetime of a decade, $K_{\mathrm{u}} \mathrm{V} / \mathrm{kT}$ must be 40 or greater. For such a particle, reducing its diameter by $25 \%$, 


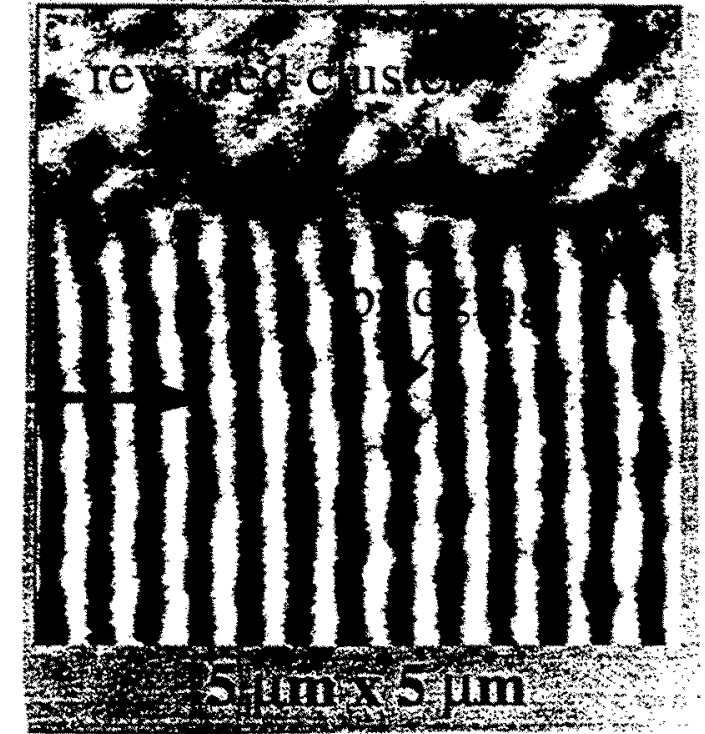

Fig. 1 MFM image of bits written on granular medium at a bit period of 5,000 flux reversal per millimeter (courtesy of J. H. Judy, U of Minn.)

therefore, its volume by a factor of two, reduces its lifetime to milliseconds, useless for data storage. Present state-of-the-art media, a CoCrX alloy, have an average grain size of approximately $120 \AA$, and are pushing this stability limit.

This article has a twofold purpose; to look at the prospects for increased bit density for magnetic recording systems on an absolute basis, and to provide a platform for comparing the futures of magnetic and of magnetooptic recording.

\section{Continuous Longitudinal Media}

The disk drive industry is based on the use of continuous, featureless, polycrystalline media, with longitudinal recording and many grains per bit. The industry has invested literally billions of dollars in developing the technology to support all components of such systems and is understandably very reluctant to abandon the investment and switch to a new storage paradigm. A number of disk drive companies have recently sponsored a study, managed by the National Storage Industry Consortium (NSIC), to analyze the future prospects of continuous longitudinal recording for very high density storage. The results of that study are confidential to the sponsoring companies, but some of the general conclusions are available.

The study group concluded that the extension of continuous longitudinal recording to $100 \mathrm{Gbit} / \mathrm{in}^{2}$ was not impossible, and a system design specifying the many parameters of such a system was generated. The design parameters are highly interrelated, and changing any one has implications for many of the other. The design parameter that we will focus on here relates to the medium and deals with grain size.

The study concluded that a grain diameter of $80 \AA$, with an appropriately adjusted anisotropy, would meet both thermal stability and signal/noise requirements. Media with an average grain size of $80 \AA$ should not be extraordinarily hard to generate, but the model puts another, very difficult to achieve, requirement on the grain size, that it be monodisperse. In Fig. 2 we show the dispersion in grain size measured at Stanford on IBM generated media with a grain size of average diameter $110 \AA$. One sees that the distribution in grain size is very wide indeed. For such a media the smaller grains will be very unstable thermally and the larger grains will contribute to degraded signal-to-noise. Zhang \& Bertram?", at UC San Diego, have calculated the effect of a dispersion in grain volume on the overall thermal stability of a film. The decay with time occurs abruptly at a long time value for the monodisperse medium but sets in earlier and is distributed over a longer time for the dispersed-size sample. The time to an $80 \%$ decay of magnetization (or signal) is four orders of magnitude less for the dispersed medium than for the monodisperse medium, reduced from 33 years to 100 seconds. That these dire predictions are correct have been verified experimentally.

The conclusion to which one is forced is that the key to achieving the desired high bit densities in longitudinal continuous media lies in generating a monodisperse polycrystalline film, not likely with present technology. In summary it would not be accurate to say that 100 Gbit/in ${ }^{2}$ can never be achieved with longitudinal continuous media, but that to achieve such a density a new technology, leading to a small (less than 10\%) dispersal in grain size, needs to be invented.

\section{Perpendicular Media}

Perpendicular media offer greater thermal stability than longitudinal media because (1) the demagnetizing fields of the bits are stabilizing rather than destabilizing, as in the case for longitudinal media and (2) the grains can be larger

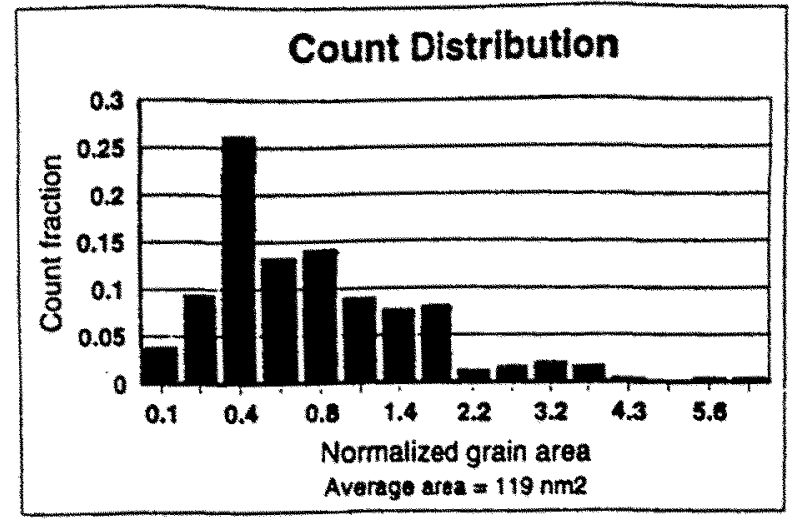

Fig. 2 Grain area distribution in high performance continuous media (media from M. Doemer, IBM, measurements N. Fussing, Stanford) 
for the same bit density because they can be columnar, normal to the film plane, with the column height not affecting the plan view grain size, important for bit density. Though there has been a great deal of study done on perpendicular recording systems, especially in Japan, no disk drive manufacturer presently uses perpendicular media, and the several companies based on this technology founded in the U.S. a decade ago have all failed. Hitachi is giving serious thought to moving to perpendicular media for very high density systems, however, none based on this technology have yet reached the market. The basic reason for the failure of perpendicular media to penetrate the market place is that, although it may offer some advantages, those advantages have not been enough to cause the magnetic recording industry to move away from the longitudinal recording technology in which it has made, over time, such a massive investment. It is likely that this situation will continue, for at least a while, even in the face of the thermal stability advantages of pattemed media.

At the recent Information Storage Technology Symposium in Kalamata, Greece, a number of scientists examined the question "What are the ultimate physical limits on the density of magnetic data storage?" The group did not consider factors such as cost or fabrication technology, but only fundamental physical constraints. The consensus was that the present continuous media longitudinal recording technology would probably not achieve densities above approximately $30 \mathrm{Gbit} / \mathrm{in}^{2}$. Further, going to perpendicular media might extend data densities using continuous granular media closer to 100 $\mathrm{Gbi} / \mathrm{in}^{2}$, but that the particle size uniformity problem might well make patterned media necessary to achieve 100 Gbit/in ${ }^{2}$. Finally, and somewhat surprising, the consensus was that, with perpendicular recording and patterned media, data densities of 1 Terrabit/in ${ }^{2}$ could be achieved consistent with acceptable thermal stability, signal/noise, and tracking requirements.

It will be very interesting to see what paths the industry will take in the future. In particular, will the factor of two or three gain in achievable densities, using continuous media, motivate the industry to change from the familiar longitudinal technology to the less well explored perpendicular technology?

\section{Patterned Media}

Patterned media may be divided conceptually into two categories. The first is similar to continuous polycrystalline media in that patterning is used to produce a regular array of small magnetic particles, similar in size to the grains of the polycrystalline medium. The recorded bit still has its shape and position deternined by the write head, but the regularity of the "grain" alloy allows gains in signal to noise and in thermal stability. The second approach is to form, usually lithographically, an array of magnetic "islands," each of which is a single magnetic domain and a single data bit. In this case the bit shape and position are built into the thin films and not determined by write head parameters.

The value of the first approach has been foreshadowed by our earlier discussion on the limitations placed on continuous longitudinal media by the non-uniformity of grain size. Various schemes for producing ordered arrays of nanoparticles have been proposed; interferometric exposure of a photo resist or self-ordering arrays such as may be formed on Langmuir-Blodgett films or with nanochannel glass fiber anrays. To date, regular dense arrays with "particle" size on the order of $100 \AA$ have not been successfully achieved. Most naturally ordering two dimensional arrays do so on a cubic or, more often, closepacked hexagonal format. Such arrays do not match well with the circular configuration typical of disk systems. Bit size in such systems will be many times particle size for good noise properties, so the problem of the superparamagnetic limits are amelionated (through the uniformity of particle size) but not pushed back significantly. No advantages in tracking are achieved.

The greatest promise for achieving very high bit high densities consistent with thermal stability appear to lie in patterned media utilizing the one island, one domain, one bit approach. The basic argument is this: for a bit of given size, a bit comprised of a single magnetic "grain" has a grain size $n$ times larger than a bit comprised of $n$ grains. The paramagnetic limit is pushed out $\mathrm{n}$-fold for the patterned medium as compared to the continuous medium, where $\mathrm{n}$ is on the order of one thousand. Patterned media therefore offer, in principle, the extension of media densities orders of magnitude beyond continuous media. In view of this great promise, why has industry not embraced the concept of patterned media? The answer is very simple: cost. Patterned media are certainly going to be more expensive to produce than continuous, featureless, media. For decades the industry has delivered ever-increasing disk capacity at no increase in cost. With patterned media, increased density will almost certainly be accompanied by increasing cost, changing the competitive position of disk memory versus other data storage techniques. Further, there are presently no viable techniques for producing patterned media with the feature size desired with an area comparable to that of a disk, even a small disk. The viability of the patterned media approach will depend on the development of fabrication technology appropriate for producing millions of disks at a reasonable cost per disk.

In the opinion of the author, the regularized multigrain approach, also sometimes called the self-organized media approach, does not offer as great an advantage for the pain of going to a patterned medium as does the lithographic, one-bit-per-island approach. Further, it offers no advantages, and perhaps even has disadvantages, for tracking and recording, whereas the one bit-per-island approach offers some significant advantages for this function. 
Within the one-island-per-bit approach there are also several branch points or options. The principal options are perpendicularly magnetized rod-shaped bits, and thin film (thickaess less than lateral dimensions) islands magnetized either longitudinally ot perpendicularly. Within these options, it is the opinion of the author that the thin film island option is the more promising though either longitudinal or perpendicular magnetization might prevail. Work on pattemed media is being pursued at several universities, and the next year or two is likely to see a significant increase in the data available on patterned media. Patterned media displaying single-domained islands or rods have already been demonstrated at Stanford, University of Minnesotas and University of California, San Diego . Near term future work at Stanford is directed at measuring the signal/noise achieved in high-density patterned arrays as compared to the signal/noise achievable with contintous media at the same densities. The signal/noise achievable in the patterned media depends primarily on the quality of the lithography, so the development of high resolution (less than $0.1 \mu \mathrm{m}$ ) lithography has high protority for patterned media. Patterned media, at least in the thin film island form, also have some significant advantages for tracking and servoing. There is increased industrial interest in patenned media, which will probably lead to considerable expansion in the research on patterned media, initially in universities but spreading ultimately to the interested companies.

\section{Conclusions}

The technology currently being used by the hard disk data storage industry is now encountering some funcamental physical barriers as it pushes toward even bigher data densities, barriers that may force a change in the present paradigm. The most pressing problem is media noise caused by the granularity of the recording medium, and the consideration of thermal stability of the particles as grains ate made smaller. Going from longitudinal to perpendicular recording may extend densities a factor of two or three beyond those achievable in the longitudinal case. Going to patterned media in principle allows data densities two order of magnitude beyond present levels. The cost of going to new technologies is a strong hamper to their adoption; in particular there is presently no viable technology for making patterned media at reasonable cost.

It is fair to say that the magnetic recording industry is going to experience turbulent times in the near future, and "business as usual" is not likely to continue.

\section{Acknowledgements}

This review was generated as part of a collaborative program between Stanford University and the Toyota Technological Institute sponsored by Grant-in-Aid for International Scientific Research (Joint Research \#09044186), The Ministry of Education, Science, Sports and Culture, Japan.

\section{References}

1) C. Tsang, T, Lin, $S$, MacDonald, M. Pinarbast, N. Robetson, H. Santini, M. Doemer, T. Reith, L. Vo, T. Diola \&. Arnett $5 \mathrm{~Gb} / \mathrm{An}^{2}$ Recording demonstration with conventional AMR dual element heads \& thin fim disk, IEEE Trans. Magn., Vol. 33, pp. 2866-2871, 1997.

2) H. Kanai, J. Kane, K. Yamada, K. Aoshima, M. Kanamine, J. Toda and Y. Mizoshita: Ni Fe/Co Fe B Spin-valve heads for over 5 Gbitin ${ }^{2}$ density recording, WeEE Trans. Magn. Vol. 33 , pp. $2872-2874,1997$

3) H.L. Hu, K. Ju, C.C. Han, D. Chabbra, Y, Guo, C. Horng: J.W. Chang, T. Torng, G. Yeh, B.B. Lal, S. Malhotra, Z. Jang, M. Yang, M. Sullivan and 1. Chao: $5 \mathrm{~Gb} / \mathrm{in}^{2}$ Recording with dual stripe magnetoresistive heads and low notse thin film disks, 1998 TMRC meeting, paper C2 (to be published in IFEE Trants. Magn. .

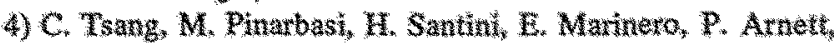
R. Olson, R. Hsiao, M. Willams, R. Payne, 3. Moore, B. Gurney, $T$, Lit and $R$, Fontatat 12 Gb/n ${ }^{2}$ Recording demonstration with $5 \mathrm{~V}$ read heads and conventional narrow pole-tip write heads, 1998 IMRC meeting, paper C3 (to be published in IEEE Trans. Magn.).

5) P.L. Lu and S.H. Charap: Thermal instability at 10 Gbit/m magnetic recording, IEEE Trans. Magn., Vol, 30 , pp. 4230 . $4232,1994$.

P.L. Lu and S.M. Charap: High density magnetic recording media design and identification; susceptibility to thermal decay, IEEE Trans. Magn. Vol, 31, pp, 2767-2769, 1995.

6) D.F. Han, 3, G. ZWu, J.K. Judy \& S.M. Srvertson: Time decay of magnetization in longtudina CoCrTa/Cr bigh density thin fitm media, IEEE Trans. Magn. Vol. 33, pp. $3025-3027,1997$.

Y. Hosoe, 1. Tamai, K. Takahashi, T. Yantanoto, T. Kanbe and Y. Yajima: Experimental study of themal decay in highdensity magnetic recording media, MEE Trans, Magn., Vol. 33, pp. 3028-3031, 1997.

Y. Hosoe, T. Kanbe, K. Takahashi, I. Tamai, S. Matsunuma and $\mathrm{Y}$. Takahashi: Thermal aftereffects in thin fim magnetic recording media, IEEE Trans. Maga. Vol. 34, pp.1528-1533, 1998.

M. Yu, M.F. Doemer \& D. . Sellmyer. Thermal stability and manostructure of $\mathrm{Co} \mathrm{Cr}$ Pt longitudinal recotoing media, IEEE Trans. Magn., Vol. 34, pp. 1534-1535, 1998.

7) 4 N Bertram: private comnunteation, to be publusted.

8) R.M.H. New, R.F.W, Pease, R.L. White, R.M. Osgood and K. Babcock: Magnetic force mictoscopy of single-domain single-crystal iron particles with miaxial surface anisotropy, 1. Appl. Pbys., Vol. 79, pp. 5851-5853, 1996.

9) S.Y. Chou, P.R. Krauss \& L. Kong: Nanolithographically defined magnetic structures and cuantum magnetic disk, $I$. Appl. Phys., Vol. 79, pp. 6101-6106, 1996.

10) R. O'Bar, M. Lederman, S. Schultz, W. Xu, A. Scheret and R.J. Tonuccit Preparation and quantitative studies of single-tomain nockel cylinders, Appl. Phys, Vol. 79, pp. $5303-5305,1996$. 